\title{
First-principles study of the interaction of hydrogen with $\operatorname{GaAs}(001)$
}

\author{
J. M. Ripalda, ${ }^{*}$ A. Khatiri, T. J. Krzyzewski, J. D. Gale, and T. S. Jones \\ Centre for Electronic Materials and Devices and Department of Chemistry, Imperial College London, \\ SW7 2AZ, London, United Kingdom
}

(Received 3 March 2003; published 29 August 2003)

\begin{abstract}
The relative stabilities of various possible structures for hydrogen terminated $\mathrm{GaAs}(001)$ surfaces have been studied at coverages ranging from 0.5 to 2.0 hydrogen atoms per surface gallium atom. We have used the local density approximation with a localized atomic orbital basis set and norm conserving pseudopotentials. The results are compared with experimental scanning tunneling microscopy images of these surfaces. We have also mapped the total energy of the system during recombinative desorption as a function of the hydrogen atomic coordinates and deduced thermal desorption rates from this data. It is concluded that hydrogen exposure of the $\mathrm{GaAs}(001)-c(4 \times 4)$ reconstructed surface leads to $\mathrm{H}$-Ga terminated surfaces with a hydrogen coverage between 0.5 and 1 hydrogen atoms per surface gallium atom and mixed $c(4 \times 2)$ and $c(2 \times 2)$ domains. Terminal and bridging hydrogen adsorption sites are identified.
\end{abstract}

DOI: 10.1103/PhysRevB.68.073311

PACS number(s): 68.43.Bc, 68.37.Ef, 68.43.Mn, 68.47.Fg

The interaction of hydrogen with semiconductor surfaces is of considerable technological interest, not only during epitaxial growth, but also as a pre-growth or post-growth surface treatment. In some cases, such as the chemical vapor deposition of diamond, atomic hydrogen plays a key role during epitaxy, which is well understood at a fundamental level. ${ }^{1}$ In other cases, such as III-V semiconductor epitaxy, the role of atomic hydrogen during growth is much less clear, and many of its possible uses have only just begun to be explored. Atomic hydrogen is often used for the removal of the native oxide and cleaning of III-V semiconductor substrates prior to epitaxial growth. ${ }^{2}$ The temperature required for this process is lower than that required for the more standard method of thermal desorption under a group V flux, and this leads to certain advantages, such as improved surface morphology immediately after cleaning. The effects of atomic hydrogen during GaAs homoepitaxy have also been studied, but various aspects remain obscure and controversial. $^{3-7}$

Surprisingly, little is known about the interaction of $\mathrm{H}$ with III-V semiconductor surfaces from a fundamental atomistic perspective. Schailey and Ray have studied the adsorption of hydrogen on GaAs clusters using the HartreeFock method, but only low hydrogen coverages were considered. ${ }^{8}$ Miyamoto and Nonoyama used the local density approximation (LDA) on a $\mathrm{GaAs}(001)$ repeating slab geometry to model H-As terminated surfaces. ${ }^{9}$ In this paper we focus on the $\mathrm{H}-\mathrm{Ga}$ interaction, since experimental evidence suggests that $\mathrm{H}$ exposure of $\mathrm{GaAs}(001)$ results in $\mathrm{H}-\mathrm{Ga}$ terminated surfaces. ${ }^{10}$ In a previous experimental study we have found that $\mathrm{H}$ exposure at temperatures between 50 and $400{ }^{\circ} \mathrm{C}$ leads to loss of surface As due to the formation of volatile arsenic hydrides, resulting in mixed $c(2 \times 2)$ and $c(4 \times 2)$ domains, with the area covered by the $c(4 \times 2)$ reconstruction increasing with temperature. ${ }^{11}$

The experiments were performed in a molecular beam epitaxy (MBE) growth chamber (DCA Instruments), equipped with in situ reflection high energy electron diffraction (RHEED) and a catalytic hydrogen cracker source (Oxford Applied). A $c(4 \times 4)$ reconstruction was stabilized on epiready, nominally flat, $n^{+}$doped $\mathrm{GaAs}(001)$ substrates which were then exposed to $480 \mathrm{~L}$ atomic hydrogen at 3 $\times 10^{-6}$ mbar pressure and a substrate temperature of $150{ }^{\circ} \mathrm{C}$. After hydrogen exposure, the samples were transferred under ultrahigh vacuum (UHV) to the scanning tunneling microscope (STM) for imaging at room temperature. A comprehensive STM study of atomic hydrogen exposed $\mathrm{GaAs}(001)$ is presented elsewhere. ${ }^{11}$

We report the results of density functional theory (DFT) calculations of various atomistic models of hydrogen terminated $\mathrm{GaAs}(001)$ surfaces, and compare the results with our experimental STM images of these surfaces. We have used the local density approximation (LDA) and the local spin density approximation (LSDA) with a basis set of localized atomic orbitals and norm-conserving pseudopotentials. Some of the calculations have been repeated using the generalized gradient approximation (GGA, Perdew-Burke-Ernzerhof functional), ${ }^{12}$ however, the obtained $\mathrm{H}$ binding energies were found to be within $4 \%$ of the results obtained with the LDA. Garcia et al. have reported that for the particular case of bulk GaAs, the use of GGA functionals leads to no systematic improvement on the accuracy of the LDA results. ${ }^{13}$ The transferability of the pseudopotentials was tested by comparison with all electron calculations for various atomic and ionic excited states. Bond lengths and band structure results obtained for bulk GaAs, InAs, GaN, and molecular hydrogen where then compared with other published LDA results as a further test of pseudopotential transferability. All calculations were performed using the SIESTA methodology, as described elsewhere. ${ }^{14}$ All key parameters where optimized in order to achieve acceptable convergence. The basis set was double $\zeta$ for $\mathrm{Ga}$ and As and triple $\zeta$ for $\mathrm{H}$, including polarization functions, and with confinement radii set so that the energy shift on the atomic orbitals (relative to the unconfined orbitals) was $<0.02 \mathrm{Ry}$. The method of Monkhorst and Pack was used to sample $k$ space at accuracy equivalent to that obtained with an $11 \AA$ radius supercell. ${ }^{15}$ The Hartree and exchange-correlation potentials were evaluated in a real space mesh with a 120 Ry equivalent plane wave energy cutoff. We have used slabs containing eight atomic layers as 


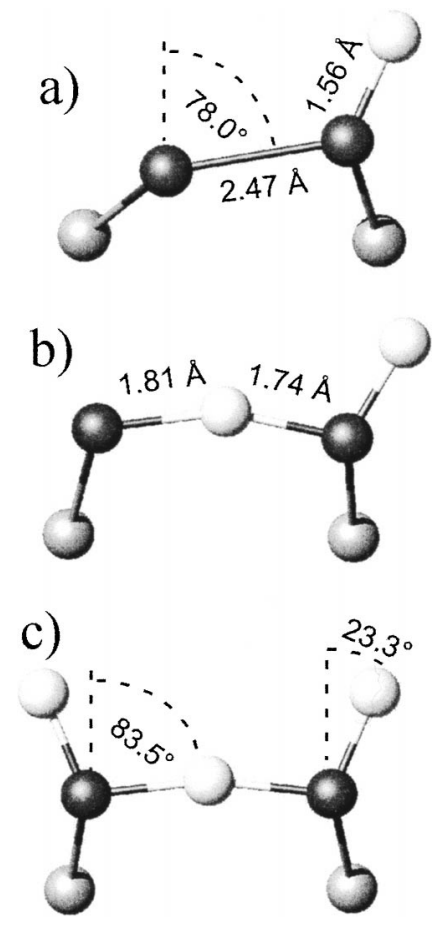

FIG. 1. Types of gallium dimer found to be stable as a function of hydrogen coverage: (a) 0.5 hydrogen atoms per surface gallium atom $(0.5 \mathrm{H} / \mathrm{Ga})$, (b) $1.0 \mathrm{H} / \mathrm{Ga}$, and (c) $1.5 \mathrm{H} / \mathrm{Ga}$. White, light gray, and dark gray atoms correspond, respectively, to hydrogen, arsenic, and gallium atoms.

a model, with the rear surface saturated with $\mathrm{H}$ atoms, which are fixed during optimization, along with the associated As layer. The use of hydrogen termination is necessary, as opposed to the use of symmetric slabs as widely utilized in other surface simulations, due to the polar nature of the (001) surface.

The geometry of various initial structures was optimized with hydrogen coverages of $0.5,1,1.5$, and 2 hydrogen atoms per surface $\mathrm{Ga}$ atom. Unit cell sizes of $(1 \times 1)$, $(1 \times 2), c(2 \times 2),(2 \times 2)$, and $c(4 \times 2)$ were considered.

Except in the saturated case, where two $\mathrm{H}$ atoms are tetrahedrally bonded to each $\mathrm{Ga}$, the top most $\mathrm{Ga}$ atoms invariably rearrange into dimers during geometry optimization, in some cases with $\mathrm{H}$ atoms bridging the gap between neighboring $\mathrm{Ga}$ atoms. Figure 1 shows the three types of Ga dimer that were found to be stable at hydrogen coverages of 0.5 , 1.0 , and 1.5 hydrogen atoms per surface gallium atom, respectively. Other types of dimer structure, such as dimers with two terminal $\mathrm{H}$ atoms and no bridging $\mathrm{H}$, or dimers with a bridging $\mathrm{H}$ and no terminal $\mathrm{H}$, were found to be unstable. The stability of the structures shown in Fig. 1 is consistent with the infrared spectroscopy results presented by Qi et $a .^{16}$ which indicate the presence of both terminal and bridging $\mathrm{H}$ atoms on hydrogen exposed $\mathrm{GaAs}(001)$.

Terminal hydrogens were always found to have a bond length of $1.56 \AA$, except at saturation (two $\mathrm{H}$ atoms per surface $\mathrm{Ga}$ ), in which case the $\mathrm{H}-\mathrm{Ga}$ bond length increased to $1.66 \AA$ A. Terminal hydrogens were never found to lie exactly aligned with the surface normal as predicted by Schailey and Ray, ${ }^{8}$ but were found at an angle to the surface normal that

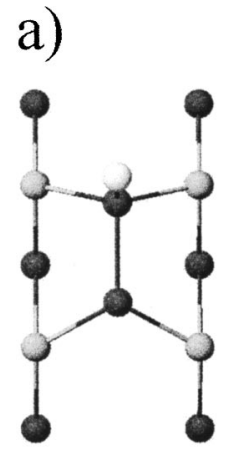

b)

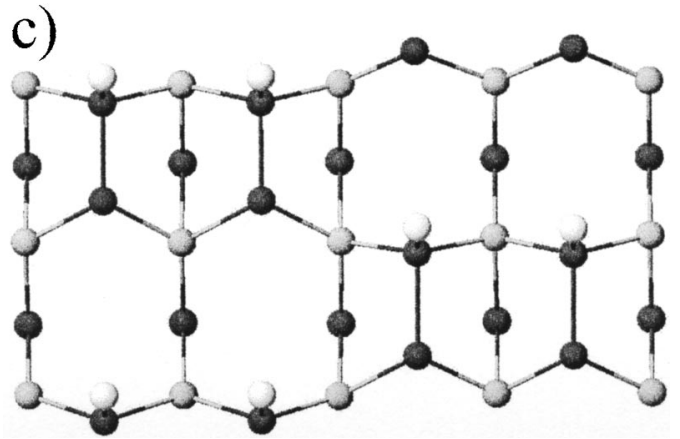

FIG. 2. Lowest energy unit cells at low coverages: (a) $(1 \times 2)$ structure at $0.5 \mathrm{H} / \mathrm{Ga}$ coverage, (b) $c(2 \times 2)$ structure at $1.0 \mathrm{H} / \mathrm{Ga}$ coverage, (c) and $c(4 \times 2)$ structure at $0.5 \mathrm{H} / \mathrm{Ga}$ coverage. White, light gray, and dark gray atoms correspond, respectively, to hydrogen, arsenic, and gallium atoms.

ranged from $21.0^{\circ}$ at a coverage of $1 \mathrm{H} / \mathrm{Ga}$, to $58.7^{\circ}$ at saturation $(2 \mathrm{H} / \mathrm{Ga})$. This discrepancy is most likely due to the fact that Schailey and Ray only considered very low H coverages without periodic boundary conditions. Bridging hydrogen atoms were found to be positioned at a distance of $1.74 \AA$ from $\mathrm{Ga}$. This distance increased to $1.81 \AA$ in the case of $\mathrm{Ga}$ atoms that were not bonded to a terminal hydrogen [Fig. 1(b)]. The bridging hydrogens lie slightly above the Ga-Ga axis as shown in Figs. 1(b) and 1(c).

Three of the surface structures that will be discussed in more detail are shown in Fig. 2. These will be referred to, respectively, as the $(1 \times 2)$ surface at a coverage of $0.5 \mathrm{H} / \mathrm{Ga}$ (a), the $c(2 \times 2)$ surface at $1 \mathrm{H} / \mathrm{Ga}$ coverage (b), and the $c(4 \times 2)$ surface at $0.5 \mathrm{H} / \mathrm{Ga}$ coverage (c). Table I summarizes the relative total energies as a function of dimer type $(\mathrm{H}$ coverage) and of dimer arrangement (unit cell symmetry). The energy differences in Table I are of the same order of magnitude as thermal energies in the $100-300^{\circ} \mathrm{C}$ range. Assuming a temperature during $\mathrm{H}$ exposure of a few hundred degrees, the results in Table I can be summarized qualita-

TABLE I. LDA Total energies (eV) per surface $\mathrm{Ga}$ atom relative to the minimum energy structure at two different hydrogen coverages.

\begin{tabular}{lccc}
\hline \hline & $(1 \times 2)$ & $c(2 \times 2)$ & $c(4 \times 2)$ \\
\hline $0.5 \mathrm{H} / \mathrm{Ga}$ & 0.000 & 0.066 & 0.021 \\
$1.0 \mathrm{H} / \mathrm{Ga}$ & 0.025 & 0.000 & 0.055 \\
\hline \hline
\end{tabular}




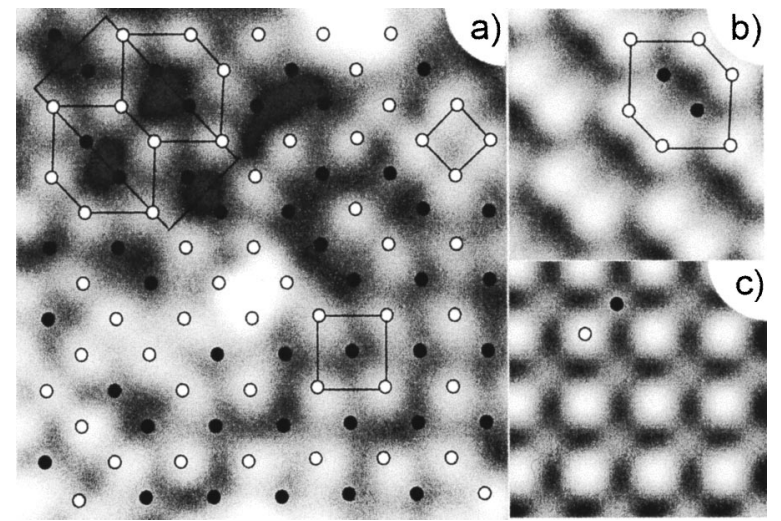

FIG. 3. (a) Experimental filled state STM image (2.8 $\times 2.8 \mathrm{~nm})$ of $\mathrm{GaAs}(001)$ exposed to $480 \mathrm{~L}$ of atomic hydrogen at $3 \times 10^{-6} \mathrm{mbar}$ and $150{ }^{\circ} \mathrm{C}$, (b) simulated STM image $(1.5$ $\times 1.5 \mathrm{~nm})$ corresponding to the $c(4 \times 2)$ structure at $0.5 \mathrm{H} / \mathrm{Ga}$ coverage, and (c) simulated STM image $(1.5 \times 1.5 \mathrm{~nm})$ corresponding to the $c(2 \times 2)$ structure at $1.0 \mathrm{H} / \mathrm{Ga}$ coverage. Ga sites occupied by a terminal $\mathrm{H}$ are highlighted with white dots, black dots indicate other $\mathrm{Ga}$ sites.

tively as follows; at $0.5 \mathrm{H} / \mathrm{Ga}$ coverage, the surface can be expected to be a mixture of $(1 \times 2)$ and $c(4 \times 2)$ domains, whereas at $1 \mathrm{H} / \mathrm{Ga}$ coverage, the surface can be expected to be a mixture of $(1 \times 2)$ and $c(2 \times 2)$ domains.

An example of a high resolution experimental STM image for the $\mathrm{GaAs}(001)$ surface exposed to $480 \mathrm{~L}$ of atomic hydrogen at $150{ }^{\circ} \mathrm{C}$ is shown in Fig. 3(a). The image was obtained at negative sample bias $(-3.6 \mathrm{~V})$ and a $0.2 \mathrm{nA}$ tunneling current. Both $c(4 \times 2)$ and $c(2 \times 2)$ repeating unit cells can be observed in Fig. 3(a) Small $(1 \times 2)$ domains $(<10$ unit cells $)$ have also been experimentally observed.

The simulated STM images in Figs. 3(b) and 3(c) have been obtained by integrating the local valence density of states in the energy range corresponding to our experimental tunneling conditions, and then plotting surfaces of constant electron density. The three lowest energy structures in Fig. 2 are compatible with our STM experimental data, although only very small domains are covered by the $(1 \times 2)$ unit cell. The energies in Table I suggest that a significant fraction of the surface should be covered by the $(1 \times 2)$ unit cell. However, our calculations model idealized perfect crystal surfaces, and in practice, a high density of point defects, adsorbates, steps, and domain boundaries will all influence the relative stabilities of the various types of local structure. Furthermore, energetics is not the only factor that determines the surface structure, and kinetic factors such as thermal desorption rates can play a key role.

So far we have dealt with the issue of what are the most stable surface structures for a given hydrogen coverage and compared these results with experimental observations. The problem of predicting theoretically the steady state hydrogen coverage under specific experimental conditions is a different one, and the correct answer is not necessarily the one that follows from thermodynamics and ground state energies. When under atomic hydrogen exposure the surface is clearly out of equilibrium, and the $\mathrm{H}$ coverage is determined by

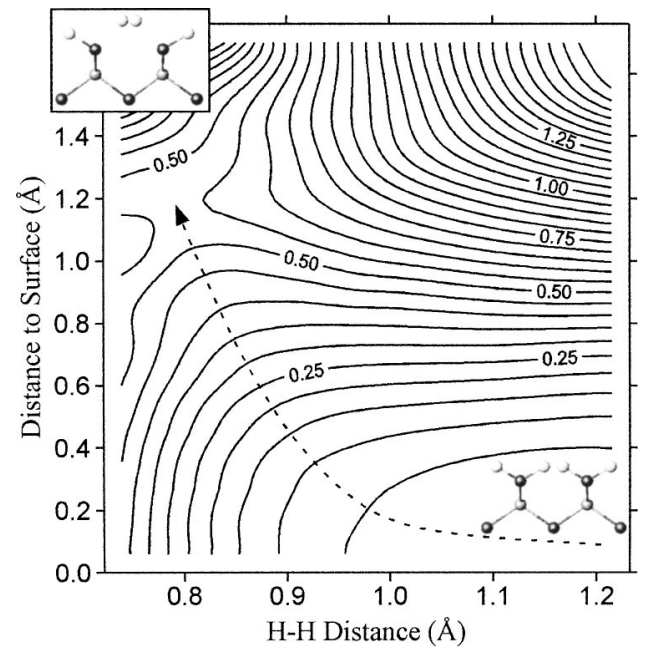

FIG. 4. Total energy (eV) as a function of hydrogen coordinates during recombinative desorption. The dashed arrow highlights the lowest energy path from the adsorption site to the saddle point on the activation barrier for desorption. The insets represent the initial and transitional structures. White, light gray, and dark gray atoms correspond, respectively, to hydrogen, arsenic, and gallium atoms.

kinetic factors. The $\mathrm{H}$ desorption rate is a function of coverage and temperature, and the steady state coverage is such that the desorption rate equals the incident $\mathrm{H}$ flux.

We have found that the adsorption of atomic hydrogen on Ga terminated $\mathrm{GaAs}(001)$ is exothermic up to saturation, with the energy released per adsorbed $\mathrm{H}$ atom depending on the adsorption site and ranging from $4.1 \mathrm{eV}$ at low hydrogen coverage to $2.6 \mathrm{eV}$ near saturation. This does not necessarily imply that the surface will saturate with hydrogen after a sufficiently long exposure, as the thermal desorption rate might become nearly equal to the incident flux as the hydrogen coverage increases.

To obtain a theoretical estimate of the hydrogen thermal desorption rate, the total energy of the system has been mapped as a function of the coordinates of the hydrogen atoms during recombinative desorption from a surface saturated with hydrogen (Fig. 4). ${ }^{17}$ The system was allowed to relax at each point in the map while the coordinates of the desorbing $\mathrm{H}$ atoms (and the back surface of the substrate) remain constrained. Between two adjacent points in the map, the coordinates of two neighboring $\mathrm{H}$ atoms were displaced symmetrically. LDA was found to overestimate the desorption barrier by $0.33 \mathrm{eV}$ relative to spin polarized GGA. The activation energy for $\mathrm{H}_{2}$ desorption in this case is $0.52 \mathrm{eV}$, which at $150^{\circ} \mathrm{C}$ implies a desorption rate of $6.4 \times 10^{6}$ desorption events per surface site per second, assuming a typical Arrhenius prefactor of $10^{13} \mathrm{~s}^{-1} \cdot{ }^{18}$ This desorption rate is six orders of magnitude faster than the hydrogen incidence rate that follows from our experimental conditions. Consequently the surface cannot attain saturation coverage, a result consistent with the STM data discussed above. Since the H binding energies are 1-2 eV higher at lower coverages, the activation energies for desorption are expected to be $1-2 \mathrm{eV}$ higher, and therefore the desorption rates should be orders of 
magnitude slower at lower $\mathrm{H}$ coverages.

In conclusion, hydrogen exposure of $\mathrm{Ga}(001)$ leads to mixed phase surfaces with both $c(4 \times 2)$ and $c(2 \times 2)$ domains. First-principles total energy calculations suggest two types of $\mathrm{H}$ adsorption sites, namely, terminal hydrogen and bridging hydrogen. The calculations show that atomic hydrogen adsorption is exothermic up to saturation $(2 \mathrm{H} / \mathrm{Ga})$, but thermal desorption rates limit the steady state coverage to $0.5-1 \mathrm{H} / \mathrm{Ga}$.

This work was supported by the EPSRC through Grant No. GR/R23367 and financial support for A.K. We would like to thank J. Neave and M. Ashwin for helpful discussions. J.M.R. acknowledges support by the Spanish government through a FPI postdoctoral grant.
*Electronic address: j.ripalda@imperial.ac.uk

†Electronic address: t.jones@imperial.ac.uk

${ }^{1}$ M. I. Heggie, G. Jungnickel, and C. D. Latham, Diamond Relat. Mater. 5, 236 (1996).

${ }^{2}$ G. R. Bell, N. S. Kaijaks, R. J. Dixon, and C. F. McConville, Surf. Sci. 401, 125 (1998).

${ }^{3}$ Y. Okada, T. Fujita, and M. Kawabe, Appl. Phys. Lett. 67, 31 (1995).

${ }^{4}$ Y. Okada and J. S. Harris, Jr., J. Vac. Sci. Technol. B 14, 1725 (1996).

${ }^{5}$ Y. Morishita, Y. Monura, S. Goto, and Y. Katatama, Appl. Phys. Lett. 67, 2500 (1995)

${ }^{6}$ R. R. La Pierre, B. J. Robinson, and D. A. Thompson, J. Cryst. Growth 191, 319 (1998).

${ }^{7}$ M. Yokozeki, H. Yonezu, T. Tsuji, K. Aizawa, and N. Oshima, Jpn. J. Appl. Phys. 37, 4626 (1998).

${ }^{8}$ R. Schailey and A. K. Ray, Comput. Mater. Sci. 22, 169 (2001).
${ }^{9}$ Y. Miyamoto and S. Nonoyama, Phys. Rev. B 46, 6915 (1992).

${ }^{10}$ J. A. Schaefer, F. Stietz, J. Woll, H. S. Wu, H. Yu, and G. J. Lapeyre, J. Vac. Sci. Technol. B 11, 1497 (1993).

${ }^{11}$ A. Khatiri, J. M. Ripalda, T. J. Krzyzewski, and T. S. Jones (unpublished).

${ }^{12}$ J. P. Perdew, K. Burke, and M. Ernzerhof, Phys. Rev. Lett. 77, 3865 (1996).

${ }^{13}$ A. García, C. Elsasser, J. Zhu, S. G. Louie, and M. L. Cohen, Phys. Rev. B 46, 9829 (1992).

${ }^{14}$ J. M. Soler, E. Artacho, J. D. Gale, A. García, J. Junquera, P. Ordejón, and D. Sánchez-Portal, J. Phys.: Condens. Matter 14, 2745 (2002).

${ }^{15}$ H. J. Monkhorst and J. D. Pack, Phys. Rev. B 13, 5188 (1976).

${ }^{16}$ H. Qi, P. E. Gee, and R. F. Hicks, Phys. Rev. Lett. 72, 250 (1994).

${ }^{17}$ A. Gross, Surf. Sci. Rep. 32, 291 (1998).

${ }^{18}$ U. Höfer, Appl. Phys. A: Mater. Sci. Process. 63, 533 (1996). 